\section{WHAT WOULD I WANT FOR MY SURGERY?}

Team-oriented. Communicative. Transparent. These are words that we want all patients to use when describing their operating teams. Teams that embody these characteristics likely work well together and make few mistakes. But creating a standardized surgical culture that encourages these qualities has proven challenging.

Implementing the surgical safety checklist can grow this culture by framing how an operating team communicates; in turn, this can minimize avoidable risks (like infections and allergic reactions) that endanger patients. It makes sense. A recent study, ${ }^{1}$ however, found no correlation between the surgical checklist and patient mortality. Does this mean that there is no value in its application?

In British Columbia, a variety of stakeholders from the surgical community have responded to this study with the hope of highlighting the value of good teamwork and communication in the operating room. There is tremendous value to the checklist beyond its statistical significance. It ensures that common objectives are being effectively communicated. It empowers all health professionals to speak up if they notice a potential error. It gives patients a voice in determining their own care.

Since there is very little education on teamwork for health professionals, past studies ${ }^{2}$ promoting the benefits of the checklist should not be discounted. Our group also suggests that hospitals invest resources and expertise to provide teams with coaching and training. This investment will undoubtedly foster the use of tools like the checklist.

The next time that you are in the operating room, imagine that it is you laying on the table. Would you want to have a high-functioning team performing your surgery? The answer is obvious. The checklist can help operating teams work better together. We hope our paper (Available at http :// bcpsqc.ca/clinical-improvement /surgical-checklist/what-would-you -want-for-your-surgery/) offers constructive ideas on how Canada's surgical community can move forward as it aims to improve care for patients.

\section{Marlies van Dijk, RN, MSc}

Director Clinical Improvement, B.C. Patient Safety \& Quality Council

Vancouver, BC

DOI: $10.1503 /$ cjs.006414

\section{References}

1. Urbach DR, Govindarajan A, Saskin R, et al. Introduction of surgical safety checklists in Ontario, Canada. $N$ Engl 7 Med 2014;370:1029-38.

2. LeBlanc J, Donnon T, Hutchison C, et al. Development of an orthopedic surgery trauma patient handover checklist. Can $\mathcal{F}$ Surg 2014;57:8-14.

\section{A CENTURY OF BREAST SURGERY: FROM RADICAL TO MINIMAL}

We read the recent article "What is the effect of screening mammography on breast cancer incidence" 1 with great interest. The introduction of breast screening programs has opened many new uncertainties on the ideal management of women with early breast cancer. This is especially true of in situ disease, as it is clear that a proportion of these women may be overtreated. We feel your readers may be interested in the history of breast cancer treatment and the changes in surgical techniques as a background for considering breast cancer incidence over time. This history is especially pertinent in light of only marginal reductions in the rate of late-stage cancer presentation, ${ }^{1}$ suggesting the screening program is unlikely to eliminate the need for more extensive surgery for later-stage presentation. In this letter we provide this historic surgical background.

In the last century, breast surgery has undergone dramatic changes in dogma; it serves as a prime example of how surgeons have made progress by challenging the limits of contemporary doctrine. The origins of breast surgery for cancer can be traced back to the 16th century. ${ }^{2}$ It was not until 1894, however, that an American surgeon, William Stewart Halsted (1852-1922), published his surgical technique for breast cancer surgery in the form of the Halsted (radical) mastectomy. ${ }^{3}$ This technique involved excising the breast, lymph nodes and pectoralis major and minor, leaving only skin covering the ribs. This extensive en bloc tissue resection resulted in considerable disfigurement and morbidity from the resultant weakened arm function and disabling lymphedema. Despite this, the vogue toward more extensive surgery continued into the midtwentieth century with a "bigger is better" approach. In fact, an American surgeon, Owen H. Wangensteen, was quoted as saying "Today, it should be said, I believe, the Halsted operation for cancer of the breast is outmoded: it is not radical enough" 4 . These newer operations involved extending the radical surgery dissection into the neck and mediastinum by supraradical mastectomy. This made no difference on patient outcomes; subsequent survival rates did not change in light of more aggressive surgery.

In 1948, Patey and Dyson (London) advocated for taking a step back with pectoralis major-sparing surgery by using modified radical mastectomy for breast cancer. The modified radical mastectomy became popular and slowly replaced more extensive surgery by the 1980s. ${ }^{5}$ Patey argued that the excision of pectoralis major did not add any significant benefit but did contribute to poor cosmetic outcomes and intraoperative blood loss. In this post- 
World War II era and particularly from the 1970s, advances in adjuvant therapy (such as hormonal, chemotherapy and radiotherapy) have been combined with less radical surgery to achieve similar survival rates compared with early more radical surgery.

The 1970s marked the age of large-scale randomized controlled trials (RCTs) assessing the extent of surgery with objective outcomes. The Alabama Breast Cancer Project, Manchester Trial, the Cardiff-St. Mary's trial and several others compared more versus less extensive surgery. In 1971, the National Surgical Adjuvant Breast and Bowel Project B-04 was initiated and was to be the largest RCT on the subject. The trial included 1079 women with clinically negative axillary nodes who underwent radical mastectomy, total mastectomy without axillary dissection but with postoperative irradiation, or total mastectomy plus axillary dissection only if their nodes became positive. There is now 25-year follow-up data from this study, which validated other studies showing no advantage from radical mastectomy. ${ }^{6}$

The true era of breast-conserving surgery is accredited to Umberto Veronesi, an Italian oncologist who progressed the idea of removing only the involved part of the breast (quadrantectomy). This was a radical idea at the time. An RCT of 701 women recruited from 1973 to 1980 and followed up for 20 years showed that the long-term survival rate among women who undergo breast-conserving surgery (with radiotherapy) was similar to that among women who undergo radical mastectomy. The evolution and minimization of breast surgery is being echoed in the field of surgery to the axilla. A number of contemporary studies have focused on the role of management of the sentinel lymph node biopsy (Z0011, Amaros, Supremo), stepping away from conventional axillary clearance.

The future of breast surgery is bright. The next decade will be marked by shifts in paradigm toward less (with narrower acceptable margins) but more focused surgery to both the breast and axilla in light of advances in newer radiotherapy and chemotherapy regimens. This is especially true with earlier stage cancer detection, which this data demonstrates. ${ }^{1}$

\section{Gurdeep Singh Mannu, MB BS, BSc,} MRCSEd

Academic Clinical Fellow, General Surgery Oxford University Hospital NHS Trust

Oxford, United Kingdom
Angeline Bhalerao, MB BS, BSc

Foundation Doctor, Academic Medicine

Norfolk and Norwich University Hospital

Norwich, United Kingdom

DOI: $10.1503 /$ cjs.005814

\section{References}

1. Latosinsky S, Boileau JF, Bryant HE, et al. CAGS and ACS evidence based reviews in surgery. 48 . What is the effect of screening mammography on breast cancer incidence? Can 7 Surg 2014;57:67-9.

2. Ekmektzoglou KA, Xanthos T, German $\mathrm{V}$, et al. Breast cancer: from the earliest times through to the end of the 20th century. Eur 7 Obstet Gynecol Reprod Biol 2009; 145:3-8.

3. Osborne MP. William Stewart Halsted: his life and contributions to surgery. Lancet Oncol 2007;8:256-65.

4. Tuttle TM, Owen H. Wangensteen, and Jerome A Urban. The pursuit of extraaxillary lymph node metastases from breast cancer. 7 Am Coll Surg 2004;199:636-43.

5. Wilson RE, Donegan WL, Mettlin C, et al. The 1982 national survey of carcinoma of the breast in the United States by the American College of Surgeons. Surg Gynecol Obstet 1984;159:309-18.

6. Fisher B, Jeong JH, Anderson S, et al. Twenty-five-year follow-up of a randomized trial comparing radical mastectomy, total mastectomy, and total mastectomy followed by irradiation. $N$ Engl $7 \mathrm{Med}$ 2002;347:567-75. 